\title{
Ganoderic Acid DM: An Alternative Agent for the Treatment of Advanced Prostate Cancer
}

\author{
Benjamin M. Johnson, Bently P. Doonan, Faisal F. Radwan and Azizul Haque* \\ Department of Microbiology and Immunology, Charles Darby Children's Research Institute, and Hollings Cancer \\ Center, Medical University of South Carolina, 173 Ashley Avenue, Charleston, SC 29425, USA
}

\begin{abstract}
Prostate cancer is the most commonly diagnosed cancer in men and accounts for significant morbidity and mortality in the western world. While traditional therapies are effective at clearing early stage cancer, they often fail to treat late stage metastatic disease. Thus, an effective therapy that targets prostate tumor growth and metastasis is desired for alleviating the disease and improving patient outcomes. Natural extracts have been the focus of recent investigation, particularly those with reduced cellular toxicity to healthy tissue. In this review, we discuss one potential candidate, ganoderic acid, an extract from the Ganoderma lucidum mushroom that has been tested in multiple cancer models. Interestingly, ganoderic acid DM (GA-DM) has shown toxicity to both androgen-dependent and independent prostate cancer cells with reduced osteoclastogenesis in late stage metastatic disease. This review will discuss the current knowledge on this GA-DM extract and the potential benefit in treating advanced prostate cancer. We will also provide an overview on the targeted delivery of GA-DM through nanoparticles that would reduce bystander toxicity and improve the drug's effectiveness. An improved understanding of this drug and its uses will advance the field of natural chemotherapeutics, particularly in treating advanced prostate cancer.
\end{abstract}

Keywords: Prostate cancer, Ganoderic acids, Osteoclastogenesis, Androgen, 5- $\alpha$-reductase, Dihydrotestosterone, Chemotherapy.

\section{INTRODUCTION}

Prostate cancer is the most commonly diagnosed cancer in men, and is the second most common cause of cancerrelated deaths in the western world [1-3]. Factors such as age, race, and geographic location all affect the likelihood of a person developing prostate cancer [4]. There are also genetic factors that make certain people more likely to develop prostate cancer. Research shows that mutations in the BRCA-2 gene, which belongs to the family of tumorsuppressor genes, may be implicated in prostate cancer [5]. Also, dietary habits may play a role in prostate cancer development. Diets rich in red meat and calcium are thought to increase the development of prostate cancer while diets incorporating vitamins $\mathrm{E}$ and $\mathrm{K}$ may decrease an individual's cancer risk [6-8]. Study also suggests that older men who exercise regularly have a much lower risk of dying from prostate cancer [9].

The prevalence and mortality associated with prostate cancer have led to a growing interest in finding more effective treatment options for the disease. Currently, when a patient is diagnosed with prostate cancer, the most common treatment options include prostatectomy, radiation, and chemotherapy [10]. Although these therapies are effective in treating most local forms of prostate cancer, many cases progress to castrate-resistant disease where alternative therapies

\footnotetext{
*Address correspondence to this author at the Department of Microbiology and Immunology, Medical University of South Carolina, 173 Ashley Avenue, BSB-201, Charleston, SC 29425, USA; Tel: 843-792-9466; Fax: 843-792-2464; E-mail: haque@musc.edu
}

are required. One of the most significant problems associated with prostate cancer is its ability to metastasize, particularly to bone [11]. Certain types of prostate cancer exploit a normal cellular process called osteoclastogenesis which leads to the formation of osteoclasts, large multinucleated cells that cause bone resorption [12]. Metastatic prostate cancer invades and colonizes this mineralized bone. Immunotherapies to combat these issues, such as vaccines and dendritic cell therapies, have been the subject of recent study and development [13-16].

While certain immunotherapeutics have shown promise in clinical trials, the search for an effective long-term treatment of later-stage prostate cancer continues, particularly one with apoptosis-inducing properties in cancer cells. One potential candidate with such therapeutic properties is ganoderic acid, a triterpene extract from the Ganoderma lucidum mushroom [17]. While the mushroom itself has long been an element of Eastern herbal medicine thought to increase vitality and life expectancy, recent studies have shown antitumor and anti-metastatic properties in a range of cancer cell types [17]. Likewise, purified extract from the G. lucidum mushroom, ganoderic acid DM (GA-DM), has been shown to be a potential candidate for reducing prostate cancer metastasis [12]. This review will focus on the factors that influence the progression of prostate cancer to its metastatic form, discuss current trends in chemo- and immunotherapeutics, and summarize the anti-tumor and anti-metastatic properties of various ganoderic acids, with a particular emphasis on current knowledge of GA-DM in treating metastatic prostate cancer. 


\section{METASTATIC PROSTATE CANCER AND OSTEO- CLASTOGENESIS}

While the majority of prostate cancer cases are treatable through conventional means, a small percentage of men who develop metastatic disease, progress to castrate-resistant prostate cancer (CRPC) [18]. This form, as the name implies, is resistant to chemical and physical castration therapies and is the cause of the vast majority of prostate cancer related deaths [18]. Another aspect of prostate cancer that leads to a significant percentage of prostate cancer-related mortality is metastasis to bone [19]. The ability of tumor cells to metastasize is a result of a variety of factors that must occur in sequence. First, the tumor cells must lose their ability to adhere to one another and also lose their cell-matrix adhesion characteristics [20]. This process coincides with the development of motility, which entails the loss of the integrity of the cadherin-catenin complex. Abnormally low levels of transmembrane proteins called cadherins and downregulation of catenins are found in a significant percentage of advanced, metastatic prostate cancer cases [20]. The most important of these is E-cadherin, and its absence leads to cell detachment and the facilitation of bone metastasis [20]. Other cadherins, like $\mathrm{N}$-cadherin and cadherin-11, have also been found in prostate cancer cells (e.g., PC-3), and are implicated in cancer cell development and metastasis [21, 22]. A study also found that the knockdown of cadherin-11 significantly reduced prostate tumor growth and bone metastasis [21]. Matrix metalloproteinases (MMPs) are also important in metastatic prostate cancer development, as they are able to degrade the extracellular matrix allowing for cancer cell invasion [20]. MMP-2 and MMP-9 are of particular importance; these have been cited as important biomarkers in prostate cancer [23]. Studies have shown higher levels of MMPs in cancer patient sera than in patients with a noncancerous condition such as benign prostatic hyperplasia (BPH) [23]. Cancer cell motility is also mediated through upregulation of Ras and Rho synthesis [20]. Ras is a vital component in regulation of cellular proliferation and invasion, whereas Rho is Ras-dependent and is a key promoter of cell motility [20]. Once the cells become motile, they are often cleared by the immune system. However, some cells are able to attach to distant sites such as bone through this migratory process. While the vast majority of prostate cancer metastases are found in bone, the activities of those metastases vary widely.

The factors responsible for the differential activity of prostate cancer metastases in bone have been the subject of recent investigation [24, 25]. It is thought that the tumor microenvironment plays a significant role in determining osteoclastic versus osteoblastic activity [24, 25]. Osteoblastinducing prostate tumors lead to excessive bone formation in a distinctive, woven pattern that results from disorganized arrangement of bone layering; this process often leads to a higher risk of bone fractures [24]. Osteoblastic responses lead to the production of type I collagen [24]. One marker of osteoblastic activity is the overexpression of procollagen (I) carboxyterminal peptide or PICP [24]. Other growth factors implicated in the development of osteoblastic responses to prostate cancer metastasis include endothelin-1, fibroblastgrowth factor 8, bone morphogenic proteins, and VEGF [24]. In addition, prostate-specific antigen (PSA), an impor- tant marker in a high percentage of prostate cancers and the basis for one of the more common prostate cancer screening techniques, has been shown to stimulate an osteoblastic response [24]. While many forms of prostate cancer induce an osteoblastic response, osteolytic prostate cancer cells exploit the natural process of bone resorption to invade mineralized bone and proliferate and expand within bone tissue [12].

The presence of an osteolytic component in prostate cancer skeletal metastases suggests that osteoclastogenesis may play a role in the establishment of these lesions $[12,26]$. Osteoclastogenesis is primarily mediated through the Receptor Activator for Nuclear Factor $\kappa$ B Ligand (RANKL)/ RANK/osteoprotegerin (OPG) system [26]. RANKL binds to RANK which is expressed on progenitor cells, and this binding causes differentiation of these cells into osteoclasts [24]. Previous studies have shown that PC-3 prostate cancer cells (notable for displaying osteolytic activity) upregulate DKK1, a Wnt antagonist that blocks proliferative effects of osteoblastic cells [27]. Studies show that PC-3 cells develop a downregulation in Smpd3, a gene with an undefined role in osteoblast development [27]. An upregulation has also been shown in expression of chemokines, such as CXCL12 and CXCL5 that have been implicated in the progression of metastatic prostate tumors. [27]. PC-3 cells also activate Rankl, which is the gene that encodes RANKL [27]. Several other factors appear to stimulate an osteoclastic response; these include IL-1, IL-6, TNF- $\alpha$, and CCL-2/MCP-1, the latter of which is a downstream chemokine activated by RANKL binding [24]. Metastasis to bone is one factor that indicates progression of prostate cancer; another involves the changing influence of androgens on prostate cancer development.

\section{ANDROGENS AND PROSTATE CANCER}

The normal development and maintenance of the prostate is dependent on androgens acting through the androgen receptor (AR) [28]. Perhaps the most important marker of prostate cancer progression is the development of androgen resistance, a process that usually occurs between fourteen and thirty months after initial androgen ablation treatment [29]. In prostate cancer cells, 5- $\alpha$-reductase plays a major role in androgen development, as it reduces testosterone to its active form, dihydrotestosterone (DHT) [30]. There are two forms of 5- $\alpha$-reductase, each encoded by different genes [31]. 5- $\alpha$-reductase 1 is found in prostatic epithelium as well as in the liver and skin while 5- $\alpha$-reductase 2 is implicated in prostate cancer and BPH [31]. DHT may bind to the AR and then translocate into the nucleus, causing an upregulation of anti-apoptotic components in prostate cancer cells [12]. One interesting component of AR activity is its impact on PSA expression [2]. PSA is an important marker for prostate cancer and a PSA screening test is one of the most common forms of early prostate cancer detection. The expression of PSA is thought to be dependent on AR activity [2]. Due to the importance of androgens in prostate cancer development, androgen ablation has been a consistent therapeutic option. However, the progression of many forms of prostate cancer to an androgen-independent form leads to the ineffectiveness of such treatments [32]. Study has shown that cadherin-11 expression is upregulated as a result of androgen ablation therapy, suggesting that androgen deprivation therapy promotes a switch to androgen-independence [33]. Another in- 
teresting result from the same study was that PC-3 cells, which naturally do not express AR, downregulated cadherin11 expression when AR was re-expressed [33]. The potent steroidal inhibitor finasteride has been used to block $5-\alpha-$ reductase activity, but side effects such as gynecomastia and myopathy as well as adverse sexual side effects have been reported with finasteride $[12,30]$. More recent studies have questioned the significance and frequency of side effects associated with finasteride, so therapeutics targeting 5- $\alpha-$ reductase activity with limited toxicity to normal cells are currently under development $[30,34]$. Another problem associated with using 5 - $\alpha$-reductase inhibitors is that in the absence of DHT, metastatic prostate cancer upregulates testosterone expression to mirror the effects of DHT on the AR [35]. In an anorchid environment, prostate cancer cells upregulate AR expression and also increase expression of enzymes involved in the conversion of androgen precursors to testosterone [35]. Concentrations of testosterone are significantly higher in metastatic prostate cancer than in earlystage prostate cancer as DHT levels decrease [35]. In addition, different prostate cancer cell types, even isolated from the same patient, can react differently to an anorchid environment, suggesting that alternatives to androgen deprivation therapy are needed [36]. Thus, novel chemotherapy, immunotherapy or a combination of chemoimmunotherapy have become popular areas of investigation.

\section{PROSTATE CANCER THERAPEUTICS}

While many different treatment options are available for patients with prostate cancer, study recommends four types of initial treatments: (a) watchful waiting, (b) surgery, (c) radiation therapy, and (d) hormone therapy [37]. As prostate cancer progresses, chemotherapy and palliative care are the most common forms of treatment [38]. In addition, patients can take part in a clinical trial if newer options become available. Prostate cancer patients may also enter clinical trials before, during, or after starting their cancer treatment. But in cases of clinical trials, better coordination with the trial investigators and follow-up tests are required. Due to the often indolent nature of prostate cancer, and because certain types of prostate cancer may be exacerbated by overtreatment, active surveillance is often used with prostate cancer patients $[10,39]$. The one obvious advantage of active surveillance over more aggressive treatment options is the avoidance of serious side effects that accompany treatments like chemotherapy, radiation, or prostatectomy [10, 37]. However, one problem observed in patients under active surveillance is increased psychological distress [10]. Radical prostatectomy is effective in a significant number of prostate cancer cases, but the significant adverse effects on sexual and urinary function cause a decrease in overall quality of life in these patients $[10,40]$. Radiotherapy and brachytherapy, two common forms of prostate cancer treatment, both lead to significant adverse side effects, including irritative voiding symptoms and incontinence that may linger years after treatment $[10,41]$. With the problems associated with traditional therapies, alternative strategies such as chemotherapy, immunotherapy, and a combination of chemoimmunotherapy have been explored.

In recent years, docetaxel has emerged as the primary chemotherapeutic agent for use in CRPC treatment [38].
Clinical trials using docetaxel showed a marginal increase in survival benefit over existing chemotherapeutics, leading to the FDA approval of docetaxel with prednisone as a first-line therapeutic [38]. However, docetaxel remains the only widely used chemotherapeutic for metastatic prostate cancer and the failure of docetaxel treatment in late-stage patients leaves few other viable treatment options [15]. Thus, combined docetaxel treatment with other therapeutics has been the subject of recent study [15]. Among the most notable treatments to be successful in clinical trials is PROVENGE ${ }^{\circledR}$ (sipuleucel-T). PROVENGE ${ }^{\circledR}$ consists of dendritic cells cocultured with a fusion protein of prostatic acid phosphatase (PAP) and GM-CSF known as PA2024 [29]. This treatment showed a 4.5 month survival improvement versus placebo in a phase III clinical trial [16]. After a second phase III trial, PROVENGE $^{\circledR}$ showed success, and was approved by the FDA [42]. It is thought that this new drug may be used in combination with chemotherapy, but the combined effect of docetaxel and PROVENGE® requires further study [43]. While all these treatments show potential benefits, the use of a natural compound with anti-tumor properties would minimize the treatment-associated risks and side effects even further. Several recent studies involving the potential antitumor effects of a methanol extract from the Ganoderma lucidum mushroom shows significant promise and a possible new direction in the field of prostate cancer therapy.

\section{GANODERIC ACIDS AS ANTICANCER THERA- PEUTICS}

The Ganoderma lucidum mushroom, also called Lingzhi in Chinese culture, has been used for thousands of years as a form of herbal medicine [17]. While this mushroom was initially thought to maintain vitality and increase life expectancy, recent research suggests that the ganoderic acid extracts from the Lingzhi mushroom may have tangible and wide-ranging medicinal benefits, most notably its toxicity to tumor cells with comparatively limited toxicity to bystander cells. Ganoderic acid extracts obtained by submerged culture showed significant dose-dependent cytotoxicity in the human BEL7402 hepatoma cell line while showing no significant cytotoxicity to a normal human cell line L02 [44]. In addition, the study showed that ganoderic acid caused cell cycle arrest at G1-S phase in the BEL7420 line, preventing the cells from moving forward toward mitosis [44].

Another study showed the effect of ganoderic acid Me (GA-Me), purified from the methanol extract of G. lucidum mycelia, on Lewis lung carcinoma (LLC) development in a C57BL/6 mouse model [45]. Following subcutaneous injection, it was found that the drug-treated mice had a significant reduction in tumor growth as well as lung metastasis, measured by the presence of nodes in lung tissue harvested after ten days [45]. Furthermore, this study showed that GA-Me treatment upregulated the activity of NK cells [45]. The drug also increased serum concentrations and mRNA expression of cytokines IL-2 and IFN- $\gamma$ along with increased NF- $\kappa B$ protein expression [45]. This suggested that NF- $\kappa \mathrm{B}$ upregulation may act as a trigger for cytokine expansion, leading to the Th1 response characterized by the upregulation of IL-2 and IFN- $\gamma$ seen in the LLC model treated with GA-Me [45]. A further study expanded on the 95-D human lung cancer cell line, supporting the former study's findings by showing 
an increase in Th1-mediated cytokine production [46]. GAMe-treated cells also downregulated MMP-2 and MMP-9 expression, suggesting its role in decreasing tumor invasion and metastasis [46]. This study also showed that GA-Me can increase tumor cell aggregation and diminish tumor cell migration and extracellular matrix adhesion [46]. However, GA-Me showed no significant cytotoxicity to 95-D cells [46]. Even at $20 \mu \mathrm{M}$ concentrations, the apoptosis induction rate was just $2.3 \%$ [46].

Another study examined the use of a different subtype of ganoderic acid, ganoderic acid T (GA-T), which was shown to exhibit better cytotoxic effects on 95-D and other tumor cell types. The $\mathrm{IC}_{50}$, or the half maximal cytotoxicity concentration, to $95-\mathrm{D}$ cells was $27.9 \mu \mathrm{g} / \mathrm{ml}$ whereas GA-T was significantly less toxic to normal cell types [47]. The study also indicated that the GA-T treatment blocked cell cycle progression at the G1-S checkpoint similarly to the study on hepatoma cells [47]. Treatment with GA-T also increased cytochrome $\mathrm{c}$ release, increased p53 and Bax expression, and decreased mitochondrial membrane potential [47]. Ganoderic acid $\mathrm{X}(\mathrm{GA}-\mathrm{X})$ was also tested in $\mathrm{HuH}-7$ hepatoma cells, where it was shown to cause apoptosis and inhibition of topoisomerase production, with the latter result leading to inhibition of DNA synthesis in GA-X-treated cells [48]. In addition, the study showed that cytochrome c, ERK and JNK kinase levels increased while the anti-apoptotic protein Bcl-2 was decreased with GA-X treatment [48].

Three more subtypes of ganoderic acid, ganoderic acids $\mathrm{A}, \mathrm{F}$, and $\mathrm{H}$ (GA-A, GA-F, and GA-H), were tested in MDA-MB-231 breast cancer cells, where a significant reduction of tumor growth was observed [49]. Breast cancer is similar to prostate cancer in terms of its developmental pattern. Like prostate cancer, breast cancer develops from a local, therapy-responsive form to a highly invasive and metastatic form that is resistant to traditional therapeutics [49]. Two factors often found to be upregulated in breast cancer

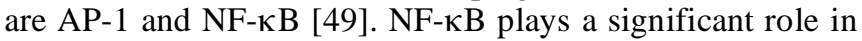
osteoclast development which is exploited by osteolytic prostate tumors. AP-1 and NF- $\mathrm{KB}$ also act in breast cancer by upregulating urokinase-type plasminogen activator (uPA), which promotes conversion of plasminogen to active plasmin, leading to cleavage of extracellular matrix components and promotion of cell adhesion and migration [49]. GA-A and GA-H showed dose-dependent cytotoxicity with an inhibition of colony formation and reduced invasiveness in the highly invasive MDA-MB-231 breast cancer cell line [49]. Table 1 summarizes the known activities of various ganoderic acid subtypes, including both anti-proliferative and anti-metastatic effects. GA-A, GA-F and GA-DM possess C3-carbonyl, GA-H and GA-X possess C3- hydroxyl, and GA-T and GA-Me possess C3-acetyl groups [49]. GA-A possesses 2 hydroxyl groups, and GA-T possesses one acetyl group in the molecule bay region [47]. GA-DM has the most hydrophobic structure and is devoid of interfering hydroxyl or acetyl side chain groups. While the effects of ganoderic acids on breast and lung cancer cell lines have been wellestablished, much less is known about its potential role as a therapeutic agent in prostate cancer. However, ganoderic acid DM is one subtype that has shown promise in attenuating advanced prostate cancer.

\section{GA-DM AS AN ALTERNATIVE CANDIDATE FOR PROSTATE CANCER THERAPY}

GA-DM is a lanostane-type triterpene isolated from the G. lucidum mushroom that induces cytotoxicity in both androgen dependent and independent prostate cancer cells [12]. Prostate cancer cell lines (PC-3 and $\mathrm{LnCaP}$ ) were shown to be inhibited by GA-DM treatment in a dose-dependent manner [12]. LnCaP cells have been shown to retain androgen dependence and maintain the presence of $\mathrm{AR}$, thus resembling early-stage prostate cancer [12]. PC-3 cells are androgen-independent and express little to no PSA, two common features of metastatic, later-stage prostate cancer [12]. Although no data exists at this time involving the use of GADM in primary prostate tumor cells, the effectiveness of the drug in these cell lines suggests that GA-DM might work in attenuating the growth of primary tumors. It has also been shown that GA-DM treatment inhibits both the activity of 5$\alpha$-reductase and the conversion of testosterone to DHT [12]. The inhibition of DHT activity possibly occurs due to the conformational similarity in the four-ringed steroidal structures of DHT and GA-DM. GA-DM competitively blocks the AR, preventing DHT binding and obstructing the normal DHT-mediated signaling pathway [12].

While the effect of GA-DM on both 5- $\alpha$-reductase and the AR mirrors that of other steroidal inhibitors, an even more promising effect of GA-DM is its inhibition of osteoclastogenesis (Fig. 1). Osteoclastogenesis is a significant factor in prostate cancer metastasis, and GA-DM has been shown to limit this process in a pre-osteoclastic cell line

Table 1. Ganoderic Acids Attenuate Malignant Growth of Various Tumor Cells

\begin{tabular}{|c|c|c|c|c|}
\hline Ganoderic Acid Subtypes & Anti-Proliferative Effects & Anti-Metastatic Effects & Cancer Cell Types & Sources \\
\hline GA-A & + & + & MDA-MDB-231 (breast) & [49] \\
\hline GA-F & - & - & MDA-MDB-231 (breast) & [49] \\
\hline GA-H & + & + & MDA-MDB-231 (breast) & [49] \\
\hline GA-Me & + & + & 95-D (lung) & {$[46]$} \\
\hline GA-T & + & - & 95-D (lung) & [47] \\
\hline GA-X & + & - & HuH-7 (hepatoma) & {$[48]$} \\
\hline GA-DM & + & + & PC-3 and LnCap (prostate) & {$[12,50]$} \\
\hline
\end{tabular}




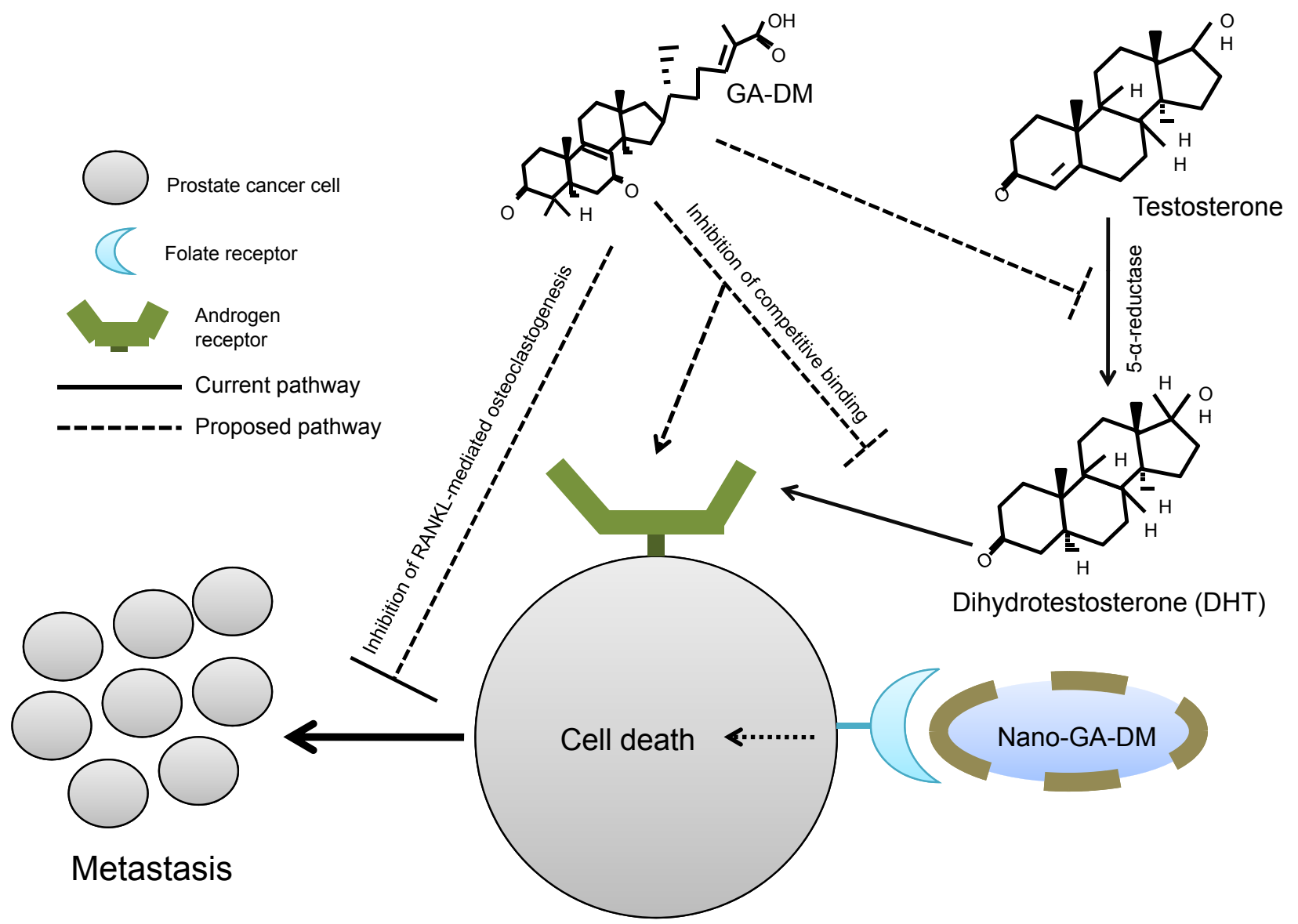

Fig. (1). Proposed mechanisms by which GA-DM may inhibit prostate cancer cell proliferation and metastasis. GA-DM (top center) inhibits 5 - $\alpha$-reductase activity, which prevents the normal conversion of testosterone to DHT (right). DHT is a potent activator of the AR, but competitive binding of GA-DM/DHT blocks DHT interaction with AR, preventing translocation to the nucleus and pro-survival signaling. GADM can also inhibit RANKL-mediated osteoclastogenesis, which reduces bone resorption, and limits bone metastasis of prostate cancer. A nanoparticle-delivered GA-DM targeted to folate receptors on the prostate cancer cell surface (bottom right) is hypothesized to facilitate cancer cell death with negligible toxicity to surrounding cells.

[12]. GA-DM treatment limited osteoclastogenesis entirely at a concentration of $25 \mu \mathrm{M}$, a concentration that left $72 \%$ of the cells viable [12]. The osteoclastogenesis-inhibiting effects of GA-DM have also been studied in the ovariectomized rat (Ovx) model as a mechanism for treating osteoporosis. This study indicated that GA-DM treatment increased bone density and decreased osteoclastogenesis, suggesting GA-DM could be a potential therapeutic alternative in prostate cancer [50]. GA-DM can act through downregulation of C-Fos and NFATc1, which also regulate osteoclastogenesis as a result of stimulation through RANKL [50].

However, a recent study showed that treatment with the G. lucidum mushroom did not affect PSA levels and only showed a slight increase in PSA doubling time [51]. It has also been shown that PSA is less effective as a marker of late-stage prostate cancer than earlier stages because of heterogeneity in prostate tumors that causes differential levels of PSA expression [52]. In addition, a study measuring survival following chemotherapy in CRPC indicated that increased median overall survival did not correlate with declines in PSA levels [52]. Furthermore, studies involving the Ganoderma lucidum mushroom did not use the purified extract but rather the entire mushroom, which could have influenced the outcome.
The proposed functions of GA-DM in various malignancies are depicted in Fig. 1. GA-DM has been shown to block 5 - $\alpha$-reductase activity, which prevents conversion of testosterone to the more active DHT. The similarity of GA-DM's structure to that of DHT allows for competitive binding of AR by GA-DM, blocking the normal signaling pathway of prostate cancer cells mediated through DHT binding to the AR. Metastasis to bone, aided by RANKL binding to the RANK receptor on osteoclast progenitor cells, is also inhibited by GA-DM. The potential use of GA-DM as an antiproliferative/metastatic agent outlined in this review imply its use in future prostate cancer disease management. However, like other chemotherapeutics, GA-DM has a downside. Direct cytotoxicity to prostate cancer cells without bystander cytotoxicity remains unattainable in GA-DM's current form. Thus, a novel prostate cancer-targeting vehicle, like a nanoparticle-conjugated delivery system, could be employed to further GA-DM's use.

\section{GA-DM AND NANOPARTICLE CONJUGATES AS FUTURE THERAPEUTICS FOR ADVANCED PROSTATE CANCER}

While GA-DM treatment reduces malignant growth and osteoclastogenesis in prostate cancer, the residual cytotoxicity to non-tumor cells remains a concern. A successful me- 
dicinal use of GA-DM will require targeted delivery in order to maximize effectiveness. Nanoparticle approaches to targeted drug delivery for malignant tumors offer new opportunity to improve patient care and quality of life by reducing off-target toxicities. Recent studies suggest that a combinatorial approach using the co-encapsulation of a lipophilic near infrared (NIR) dye and an anticancer drug within hydrophobic pockets in the polymeric matrix of poly(acrylic acid) (PAA)-coated IONPs (PAA-IONPs) could be employed for combined optical imaging, magnetic resonance imaging (MRI) detection, and targeted cancer therapy [53-58]. These studies used water-based and green chemistry approaches to synthesize nanoparticles that had five key components: (a) an encapsulated chemotherapeutic agent (Taxol) for cancer therapy, (b) surface functionality (folic acid ligand) for cancer targeting, (c) click-chemistry-based conjugation of targeting ligands, (d) an encapsulated NIR dye for fluorescent imaging capabilities, and (e) a superparamagnetic iron oxide core for MRI. The taxol-carrying nanoparticles have also been modified so that they could carry the drug only to the cancer cells, allowing targeted cancer treatment without harming healthy cells [56]. This was achieved by attaching a vitamin (folic acid) derivative that cancer cells preferentially consume in high amounts. Because the nanoparticles also carry a fluorescent dye and an iron oxide magnetic core, their locations within the cells and the body can be seen by optical imaging and MRI, which would allow a physician to see how the tumor is responding to the treatment. The nanoparticles can also be engineered without the drug and used as imaging (contrast) agents for cancer. If there is no cancer, the biodegradable nanoparticles will not bind to the tissue and will be eliminated by the liver. Studies suggest that folate receptors are overexpressed in a variety of cancers including prostate cancer, but not in normal cells [59]. Utilizing a combinatorial approach, future study may synthesize GA-DM-nanoparticle conjugates to investigate the application that this treatment could have in advanced prostate cancer.

\section{CONCLUSIONS}

Prostate cancer is a major cause of death among men in the western world. Current treatment and therapeutic options are beneficial to early stage patients but lack significant benefit to metastatic, late-stage patients. Prostate cancer preferentially metastasizes to bone, exploiting the normal cellular processes of bone formation and resorption to invade and colonize bone cells. Another point of emphasis in prostate cancer therapy is the targeting of androgens. Both 5- $\alpha$ reductase and the AR play crucial roles in prostate cancer development. 5- $\alpha$-reductase catalyzes testosterone to DHT, and then binds to the AR, promoting cell survival and proliferation. Based on these factors, research involving immunotherapy, chemoimmunotherapy, and even herbal therapeutics is of great importance and interest. While many immunotherapeutic and chemotherapeutic agents have been studied in clinical trials, finding an agent with significant toxicity to tumor cells and limited toxicity to bystander cells remains a challenge. G. lucidum mushroom extracts are cytotoxic to tumor cells with some GA subtypes having anti-metastatic properties. While extensive work has been performed using GA subtypes in breast and lung cancers, the effects of GA subtypes on prostate cancer are less clear. Studies involving GA-DM are of particular interest because GA-DM treatment has shown cytotoxicity in prostate cancer cells regardless of their dependence on androgens. GA-DM has been shown to limit the conversion of testosterone to DHT and prevent DHT binding to the androgen receptor through competitive inhibition. GA-DM has also been implicated in the inhibition of osteoclastogenesis, a process exploited by certain prostate cancer cells to promote bone metastasis. While GA-DM remains promising, introduction of GA-DMinfused-nanoparticles with targeted delivery to malignant cells could be an alternative approach to combat advanced prostate cancer. Taken together, this review suggests the potential benefits of GA-DM in treating advanced metastatic prostate cancer.

$\begin{array}{lll}\text { ABBREVIATIONS } & \\ \text { GA } & = & \text { Ganoderic acid } \\ \text { MMP } & = & \text { Matrix metallonproteinase } \\ \text { CRPC } & = & \text { Castrate resistant prostate cancer } \\ \text { BPH } & = & \text { Benign prostatic hyperplasia } \\ \text { RANK } & = & \text { Receptor factor for nuclear factor } \kappa \text { B } \\ \text { VEGF } & = & \text { Vascular endothelial growth factor } \\ \text { PSA } & = & \text { Prostate Specific Antigen } \\ \text { DHT } & = & \text { Dihydrotestosterone } \\ \text { AR } & = & \text { Androgen receptor } \\ \text { IONP } & = & \text { Ion oxide nanoparticle }\end{array}$

\section{ACKNOWLEDGEMENTS}

This work was supported by grants from the National Institutes of Health (CA129560 and CA129560-S1) to A. Haque. The authors also wish to acknowledge Dr. Jason God for reviewing the manuscript.

\section{REFERENCES}

[1] Lassi K, Dawson NA. Update on castrate-resistant prostate cancer: 2010. Curr Opin Oncol 2010; 22(3): 263-7.

[2] Jiang J, Huang H. Targeting the androgen receptor by taxol in castration-resistant prostate cancer. Mol Cell Pharmacol 2010; 2(1): $1-5$.

[3] Scosyrev E, Messing J, Noyes K, Veazie P, Messing E. Surveillance Epidemiology and End Results (SEER) program and population-based research in urologic oncology: an overview. Urol Oncol 2010. [Epub ahead of print].

[4] Bostwick DG, Burke HB, Djakiew D, et al. Human prostate cancer risk factors. Cancer 2004; 101(10 Suppl): 2371-490.

[5] Schmidt U, Fiedler U, Pilarsky CP, et al. Identification of a novel gene on chromosome 13 between BRCA-2 and RB-1. Prostate 2001; 47(2): 91-101.

[6] Nimptsch K, Rohrmann S, Kaaks R, Linseisen J. Dietary vitamin K intake in relation to cancer incidence and mortality: results from the Heidelberg cohort of the European Prospective Investigation into Cancer and Nutrition (EPIC-Heidelberg). Am J Clin Nutr 2010; 91(5): 1348-58.

[7] Ma RW, Chapman K. A systematic review of the effect of diet in prostate cancer prevention and treatment. J Hum Nutr Diet 2009; 22(3): 187-99; quiz 200-2. 
[8] Sinha R, Park Y, Graubard BI, et al. Meat and meat-related compounds and risk of prostate cancer in a large prospective cohort study in the United States. Am J Epidemiol 2009; 170(9): 1165-77.

[9] Patel AV, Rodriguez C, Jacobs EJ, et al. Recreational physical activity and risk of prostate cancer in a large cohort of U.S. men. Cancer Epidemiol Biomarkers Prev 2005; 14(1): 275-9.

[10] Singh J, Trabulsi EJ, Gomella LG. The quality-of-life impact of prostate cancer treatments. Curr Urol Rep 2010; 11(3): 139-46.

[11] Coleman RE, Lipton A, Roodman GD, et al. Metastasis and bone loss: advancing treatment and prevention. Cancer Treat Rev 2010.

[12] Liu J, Shiono J, Shimizu K, et al. Ganoderic acid DM: antiandrogenic osteoclastogenesis inhibitor. Bioorg Med Chem Lett 2009; 19(8): 2154-7.

[13] Zarour L, Alumkal J. Emerging therapies in castrate-resistant prostate cancer. Curr Urol Rep 2010; 11(3): 152-8.

[14] Di Lorenzo G, Buonerba C, Autorino R, De Placido S, Sternberg CN. Castration-resistant prostate cancer: current and emerging treatment strategies. Drugs 2010; 70(8): 983-1000.

[15] Galsky MD, Vogelzang NJ. Docetaxel-based combination therapy for castration-resistant prostate cancer. Ann Oncol 2010. [Epub ahead of print].

[16] Madan RA, Mohebtash M, Schlom J, Gulley JL. Therapeutic vaccines in metastatic castration-resistant prostate cancer: principles in clinical trial design. Expert Opin Biol Ther 2010; 10(1): 19-28.

[17] Yuen JW, Gohel MD. Anticancer effects of Ganoderma lucidum: a review of scientific evidence. Nutr Cancer 2005; 53(1): 11-7.

[18] Hofland J, van Weerden WM, Dits NF, et al. Evidence of limited contributions for intratumoral steroidogenesis in prostate cancer. Cancer Res 2010; 70(3): 1256-64.

[19] Lipton A. Bone continuum of cancer. Am J Clin Oncol 2010; 33(3 Suppl): S1-7.

[20] Clarke NW, Hart CA, Brown MD. Molecular mechanisms of metastasis in prostate cancer. Asian J Androl 2009; 11(1): 57-67.

[21] Huang CF, Lira C, Chu K, et al. Cadherin-11 increases migration and invasion of prostate cancer cells and enhances their interaction with osteoblasts. Cancer Res 2010; 70(11): 4580-9.

[22] Jennbacken $\mathrm{K}$, Tesan T, Wang W, et al. N-cadherin increases after androgen deprivation and is associated with metastasis in prostate cancer. Endocr Relat Cancer 2010; 17(2): 469-79.

[23] Di Carlo A, Mariano A, Terracciano D, et al. Matrix metalloproteinase-2 and -9 in the urine of prostate cancer patients. Oncol Rep 2010; 24(1): 3-8.

[24] Morrissey C, Vessella RL. The role of tumor microenvironment in prostate cancer bone metastasis. J Cell Biochem 2007; 101(4): 87386

[25] Li Y, Sikes RA, Malaeb BS, et al. Osteoblasts can stimulate prostate cancer growth and transcriptionally down-regulate PSA expression in cell line models. Urol Oncol 2010. [Epub ahead of print].

[26] Roato I, D'Amelio P, Gorassini E, et al. Osteoclasts are active in bone forming metastases of prostate cancer patients. PLoS One 2008; 3(11): e3627.

[27] Schulze J, Albers J, Baranowsky A, et al. Osteolytic prostate cancer cells induce the expression of specific cytokines in boneforming osteoblasts through a Stat3/5-dependent mechanism. Bone 2010; 46(2): 524-33

[28] Patrao MT, Silva EJ, Avellar MC. Androgens and the male reproductive tract: an overview of classical roles and current perspectives. Arq Bras Endocrinol Metabol 2009; 53(8): 93445 .

[29] Risk M, Corman JM. The role of immunotherapy in prostate cancer: an overview of current approaches in development. Rev Urol 2009; 11(1): 16-27.

[30] Liu J, Kurashiki K, Shimizu K, Kondo R. Structure-activity relationship for inhibition of 5alpha-reductase by triterpenoids isolated from Ganoderma lucidum. Bioorg Med Chem 2006; 14(24): p. 8654-60.

[31] Bratoeff E, Garcia P, Heuze Y, et al. Molecular interactions of progesterone derivatives with 5 alpha-reductase types 1 and 2 and androgen receptors. Steroids 2010; 75(7): 499-505.
[32] Niu Y, Chang TM, Yeh S, et al. Differential androgen receptor signals in different cells explain why androgen-deprivation therapy of prostate cancer fails. Oncogene 2010; 29(25): 3593-604.

[33] Lee YC, Cheng CJ, Huang M, et al. Androgen depletion upregulates cadherin-11 expression in prostate cancer. J Pathol 2010; 221(1): 68-76.

[34] Vickers AJ, Savage CJ, Lilja H. Finasteride to prevent prostate cancer: should all men or only a high-risk subgroup be treated? J Clin Oncol 2010; 28(7): 1112-6.

[35] Montgomery RB, Mostaghel EA, Vessella R, et al. Maintenance of intratumoral androgens in metastatic prostate cancer: a mechanism for castration-resistant tumor growth. Cancer Res 2008; 68(11): 4447-54.

[36] Arlen PM, Gulley JL. Therapeutic vaccines for prostate cancer: a review of clinical data. Curr Opin Investig Drugs 2005; 6(6): 592 6.

[37] Zeliadt SB, Moinpour CM, Blough DK, et al. Preliminary treatment considerations among men with newly diagnosed prostate cancer. Am J Manag Care 2010; 16(5): e121-30.

[38] Singh P, Yam M, Russell PJ, Khatri A. Molecular and traditional chemotherapy: a united front against prostate cancer. Cancer Lett 2010; 293(1): 1-14.

[39] Tareen B, Godoy G, Taneja SS. Focal therapy: a new paradigm for the treatment of prostate cancer. Rev Urol 2009; 11(4): 203-12.

[40] Mina DS, Matthew AG, Trachtenberg J, et al. Physical activity and quality of life after radical prostatectomy. Can Urol Assoc J 2010; 4(3): 180-6.

[41] Phan J, Swanson DA, Levy LB, et al. Late rectal complications after prostate brachytherapy for localized prostate cancer: incidence and management. Cancer 2009; 115(9): 1827-39.

[42] Schrijvers D, Van Erps P, Cortvriend J. Castration-refractory prostate cancer: new drugs in the pipeline. Adv Ther 2010; 27(5): 285-96.

[43] Higano CS, Small EJ, Schellhammer P, et al. Sipuleucel-T. Nat Rev Drug Discov 2010; 9(7): 513-4.

[44] Yang HL. Ganoderic acid produced from submerged culture of Ganoderma lucidum induces cell cycle arrest and cytotoxicity in human hepatoma cell line BEL7402. Biotechnol Lett 2005; 27(12): 835-8.

[45] Wang G, Zhao J, Liu J, et al. Enhancement of IL-2 and IFNgamma expression and NK cells activity involved in the anti-tumor effect of ganoderic acid Me in vivo. Int Imunopharmacol 2007 7(6): 864-70

[46] Chen NH, Liu JW, Zhong JJ. Ganoderic acid Me inhibits tumor invasion through down-regulating matrix metalloproteinases 2/9 gene expression. J Pharmacol Sci 2008; 108(2): 212-6.

[47] Tang W, Liu JW, Zhao WM, Wei DZ, Zhong JJ. Ganoderic acid T from Ganoderma lucidum mycelia induces mitochondria mediated apoptosis in lung cancer cells. Life Sci 2006; 80(3): 205-11.

[48] Li CH, Chen PY, Chang UM, et al. Ganoderic acid X, a lanostanoid triterpene, inhibits topoisomerases and induces apoptosis of cancer cells. Life Sci 2005; 77(3): 252-65.

[49] Jiang J, Grieb B, Thyagarajan A, Sliva D. Ganoderic acids suppress growth and invasive behavior of breast cancer cells by modulating AP-1 and NF-kappaB signaling. Int J Mol Med 2008; 21(5): 577 84.

[50] Miyamoto I, Liu J, Shimizu K, et al. Regulation of osteoclastogenesis by ganoderic acid DM isolated from Ganoderma lucidum. Eur J Pharmacol 2009; 602(1): 1-7.

[51] Yoshimura K, Kamoto T, Ogawa O, et al. Medical mushrooms used for biochemical failure after radical treatment for prostate cancer: an open-label study. Int J Urol 2010; 17(6): 548-54.

[52] Payne H, Cornford P. Prostate-specific antigen: An evolving role in diagnosis, monitoring, and treatment evaluation in prostate cancer. Urol Oncol 2010. [Epub ahead of print].

[53] Josephson L, Kircher MF, Mahmood U, Tang Y, Weissleder R. Near-infrared fluorescent nanoparticles as combined MR/optical imaging probes. Bioconjug Chem 2002; 13(3): 554-60.

[54] Nasongkla N, Bey E, Ren J, et al. Multifunctional polymeric micelles as cancer-targeted, MRI-ultrasensitive drug delivery systems. Nano Lett 2006; 6(11): 2427-30. 
[55] Weng KC, Noble CO, Papahadjopoulos-Sternberg B, et al. Targeted tumor cell internalization and imaging of multifunctional quantum dot-conjugated immunoliposomes in vitro and in vivo. Nano Lett 2008; 8(9): 2851-7.

[56] Santra S, Kaittanis C, Grimm J, Perez JM. Drug/dye-loaded, multifunctional iron oxide nanoparticles for combined targeted cancer therapy and dual optical/magnetic resonance imaging. Small 2009; 5(16): 1862-8.

[57] Santra S, Kaittanis C, Perez JM. Aliphatic hyperbranched polyester: a new building block in the construction of multi- functional nanoparticles and nanocomposites. Langmuir 2010; 26(8): 5364-73.

[58] McCarthy JR, Kelly KA, Sun EY, Weissleder R. Targeted delivery of multifunctional magnetic nanoparticles. Nanomedicine (Lond) 2007; 2(2): 153-67.

[59] Hattori Y, Maitani Y. Folate-linked nanoparticle-mediated suicide gene therapy in human prostate cancer and nasopharyngeal cancer with herpes simplex virus thymidine kinase. Cancer Gene Ther 2005; 12(10): 796-809.

(C) Johnson et al.; Licensee Bentham Open.

This is an open access article licensed under the terms of the Creative Commons Attribution Non-Commercial License (http://creativecommons.org/licenses/ by-nc/3.0/) which permits unrestricted, non-commercial use, distribution and reproduction in any medium, provided the work is properly cited. 